\title{
Composição de espécies de Arctiidae (Lepidoptera) no sul do Brasil: relação entre tipos de vegetação e entre a configuração espacial do hábitat
}

\author{
Viviane Gianluppi Ferro ${ }^{1} \&$ José Augusto Teston ${ }^{2}$
}

${ }^{1}$ Departamento de Zoologia, Laboratório de Ecologia de Insetos, Instituto de Biociências, Universidade Federal do Rio Grande do Sul. Av. Bento Gonçalves 9500, Bloco IV, Prédio 43435, 91501-970 Porto Alegre-RS, Brasil. vgferro@ yahoo.com

${ }^{2}$ Faculdade de Ciências Biológicas, Universidade Federal do Pará, Campus Universitário de Altamira. Rua Coronel José Porfírio 2515, 68372-040 Altamira-PA, Brasil. jateston@ufpa.br

\begin{abstract}
Composition of the Arctiidae species (Lepidoptera) in southern Brazil: relationship among vegetation types and among habitat spatial configuration. We describe the beta-diversity pattern of the Arctiidae in Rio Grande do Sul State (RS) and assess whether this pattern is related to similarities in vegetation type or geographic distance among areas. We analysed 9420 specimens in 13 scientific collections, as well as two published checklists. Three hundred twenty nine arctiid species were recorded for 55 localities. This richness corresponded to $5.6 \%$ of the Neotropical fauna, and $16.5 \%$ of the estimated Brazilian fauna. Fifty two species (15.8\%) were recorded for the first time in the state. There was no relationship between beta-diversity (Sorensen distance) and geographic distance among the localities, suggesting that the spatial configuration of the habitat did not influence significantly the locomotion of the Arctiidae moths among landscapes. The multivariate analysis suggested that the Arctiidae fauna differ among vegetation types. The composition of the fauna at the Floresta Ombrófila Mista (Araucaria forest) is very distinct from other vegetation type faunas. Species richness was higher on forest habitats than in open habitats.
\end{abstract}

KEYWORDS. Beta-diversity; Lithosiinae; Neotropical fauna; vegetation types.

RESUMO. Composição de espécies de Arctiidae (Lepidoptera) no sul do Brasil: relação entre tipos de vegetação e entre a configuração espacial do hábitat. Este trabalho descreve o padrão de diversidade beta das mariposas Arctiidae no Estado do Rio Grande do Sul (RS) e avalia se esse padrão é relacionado com o tipo de vegetação ou com a distância geográfica entre as áreas. A partir da observação de 9420 espécimes depositados em 13 coleções científicas e de duas listas publicadas na literatura, obteve-se registro de 329 espécies de arctí́deos em 55 localidades do RS. Essa riqueza corresponde a 5,6\% da fauna Neotropical e 16,5\% da fauna estimada para o Brasil. Cinqüenta e duas espécies $(15,8 \%)$ foram registradas pela primeira vez no Estado. Não houve relação entre a diversidade beta (distância de Sorensen) e a distância geográfica entre as localidades, sugerindo que a configuração espacial do ambiente não influencia de forma significativa a locomoção das mariposas Arctiidae entre as paisagens. As análises multivariadas indicaram que a fauna de Arctiidae apresenta uma composição diferente em cada tipo de vegetação. A composição da fauna de áreas de Floresta Ombrófila Mista (Mata de Araucária) difere da fauna dos demais tipos de vegetação. Além disso, verificou-se uma maior riqueza de espécies em ambientes florestais do que em campestres.

PALAVRAS-CHAVE. Diversidade beta; fauna Neotropical; tipo de vegetação; Lithosiinae.

A diversidade beta, isto é, a variação na composição de espécies entre sítios (Whittaker 1972), tem um importante papel na biologia da conservação (Blackburn \& Gaston 1996). Caso a composição de espécies de um determinado local não mude com a distância, a localização de novas unidades de conservação dentro deste local não é importante. Entretanto, se existem diferenças na composição de espécies, são necessários estudos sobre a fauna de vários sítios dentro deste local para assegurar que as áreas destinadas a futuras reservas protejam o maior número de espécies possível (Bridgewater et al. 2004).

A similaridade de espécies entre dois sítios geralmente possui uma relação negativa com a distância geográfica dos sítios, ou seja, sítios mais distantes tendem a apresentar uma composição de espécies menos similar (e.g. Whittaker 1972). Contudo, poucos estudos avaliam os fatores que determinam esse decréscimo da similaridade com a distância. Nekola \&
White (1999) sugeriram que os principais fatores determinantes deste decréscimo seriam as diferenças ambientais dos nichos e as restrições espaço-temporais das espécies. As diferenças dos nichos produzem um decréscimo da similaridade das espécies com a distância geográfica porque sítios vizinhos tendem a ser mais similares em suas condições ambientais do que sítios distantes (Legendre 1993). Já as restrições espaçotemporais produzem um decréscimo na similaridade das espécies com a distância geográfica porque a configuração espacial (tamanho e isolamento de hábitats e características da matriz que circunda o hábitat, por exemplo) influencia a locomoção de espécies entre paisagens (Nekola \& White 1999).

O Estado do Rio Grande do Sul (RS), dada sua localização na transição entre as regiões tropical e temperada, apresenta características peculiares em sua fauna, uma vez que engloba elementos de ambas as regiões. Todavia, há ainda muitas lacunas nas informações sobre a fauna desse Estado, e as que 
existem são, na maioria, incompletas e de difícil acesso (Romanowski \& Buss 1997). A princípio, supõe-se que os diferentes tipos de vegetação do RS devam apresentar faunas com composições distintas. Contudo, a veracidade desse pressuposto ainda precisa ser investigada.

Este trabalho descreve o padrão de diversidade beta de Arctiidae (Lepidoptera) no RS e avalia se esse padrão é mais fortemente relacionado com o tipo de vegetação ou com a distância geográfica entre as áreas. A família Arctiidae foi selecionada para este estudo pelos seguintes motivos: (1) é um táxon com grande número de espécies (duas mil espécies estimadas para o Brasil segundo Brown Jr. \& Freitas 1999), (2) é bem representada nas coleções brasileiras, (3) sua taxonomia é relativamente bem resolvida, (4) é um dos grupos de lepidópteros noturnos mais utilizados como bioindicadores ambientais (Hilty \& Merenlender 2000) e (5) foi intensivamente estudada no RS nos últimos anos (Teston \& Corseuil 2002, 2003a, 2003b, 2004; Teston et al. 2006).

Baseados em dados de museus e coletas, Teston \& Corseuil (2002, 2003a, 2003b) listaram 325 espécies para o Estado do RS (25 espécies da tribo Pericopini, 31 de Arctiini, uma de Callimorphini, 129 de Phaegopterini, 79 de Ctenuchini e 60 de Euchromiini). No entanto, essas listas incluem apenas as espécies da subfamília Arctiinae e os autores não citam as localidades onde cada espécie foi registrada. Biezanko (1983, 1985) também publicou listas de Arctiidae ocorrentes no RS, mas apenas para a zona sudeste e noroeste do Estado, coletando 257 espécies. Esse último autor foi o único pesquisador recente que publicou dados sobre os Lithosiinae do RS, registrando 32 espécies para o Estado. Apesar do esforço em conhecer a composição de espécies de arctí́deos que ocorrem no RS, não existem trabalhos que descrevam os padrões de diversidade beta dessas mariposas em escalas regionais no sul do Brasil.

\section{MATERIALE MÉTODOS}

Os dados sobre a distribuição das mariposas Arctiidae no RS foram obtidos, predominantemente, de 9420 espécimes depositados em 13 coleções entomológicas brasileiras. São elas: (1) Coleção Becker (Camacan, BA), (2) Coleção do Centro de Pesquisa Agropecuária de Clima Temperado da Embrapa, (3) Museu Entomológico Ceslau Biezanko da Universidade Federal de Pelotas e (4) Museu de Ciências da Universidade Católica de Pelotas (ambos em Pelotas, RS), (5) Coleção Entomológica Padre Jesus Santiago Moure da Universidade Federal do Paraná (Curitiba, PR), (6) Museu de Zoologia da Universidade de São Paulo (São Paulo, SP), (7) Museu de Ciências Naturais da Fundação Zoobotânica do Rio Grande do Sul, (8) Museu Ramiro Gomes Costa da Fundação Estadual de Pesquisa Agropecuária, (9) Coleção do Setor de Entomologia da Faculdade de Agronomia da Universidade Federal do Rio Grande do Sul e (10) Museu de Ciências e Tecnologia da Pontifícia Universidade Católica do Rio Grande do Sul (ambos em Porto Alegre, RS), (11) Coleção Entomológica do Instituto Oswaldo Cruz e (12) Museu Nacional da
Universidade Federal do Rio de Janeiro (ambos no Rio de Janeiro, RJ) e (13) Coleção particular do segundo autor (Iraí, $\mathrm{RS})$. Durante as visitas, foram registrados os dados de coleta (localidade, data, altitude e coordenadas geográficas) e os dados de identificação (gênero e espécie) de todos os exemplares de Arctiidae com ocorrência para o RS. Os indivíduos com identificação e proveniência duvidosas não foram considerados. Para a maioria dos espécimes, as coordenadas geográficas e altitudes das localidades foram obtidas através do sistema de informação "splink" desenvolvido pelo Centro de Referência em Informação Ambiental (http://splink.cria.org.br). Também foram utilizadas listas de espécies de arctiídeos já publicadas (Teston \& Corseuil 2004; Teston et al. 2006). Os dados de ocorrência das espécies no Estado foram inseridos num programa de banco de dados (Microsoft Access) para análise posterior.

Com o objetivo de obter uma caracterização das semelhanças entre as faunas das localidades, foram feitas análises de classificação aglomerativa (Unweighted PairGroups Method using Arithmetic Averages - UPGMA) combinando a fauna por localidade e, também, por tipo de vegetação (Floresta Ombrófila Mista, Floresta Estacional Decidual, Floresta Estacional Semidecidual, Estepe, Área de Tensão Ecológica e Área de Formação Pioneira). As localidades foram classificadas quanto ao seu tipo de vegetação a partir do Mapa de Vegetação do Brasil (IBGE 2004). Estas análises foram feitas apenas com as localidades cuja riqueza de Arctiidae foi superior a 20 espécies para diminuir o ruído das análises pela inclusão de localidades extremamente subamostradas.

Além disso, foi feito um teste de Mantel para verificar a existência de correlação entre a diversidade beta (medida como distância de Sorensen, com dados de presença e ausência) e a

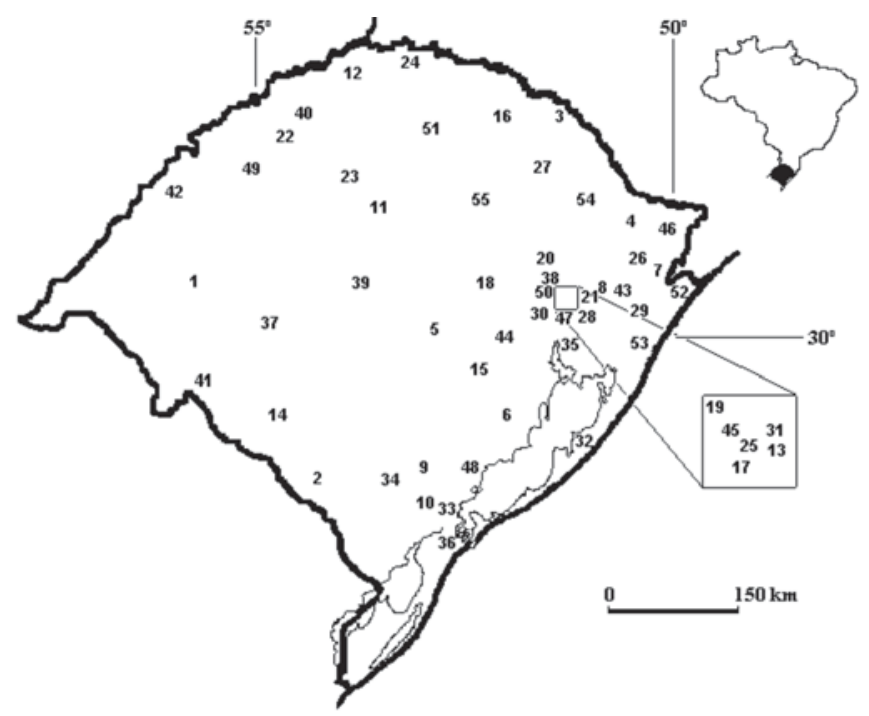

Fig. 1. Mapa do Estado do Rio Grande do Sul indicando a posição dos municípios com registro de coleta de Arctiidae. Os números correspondem aos da Tabela I. 


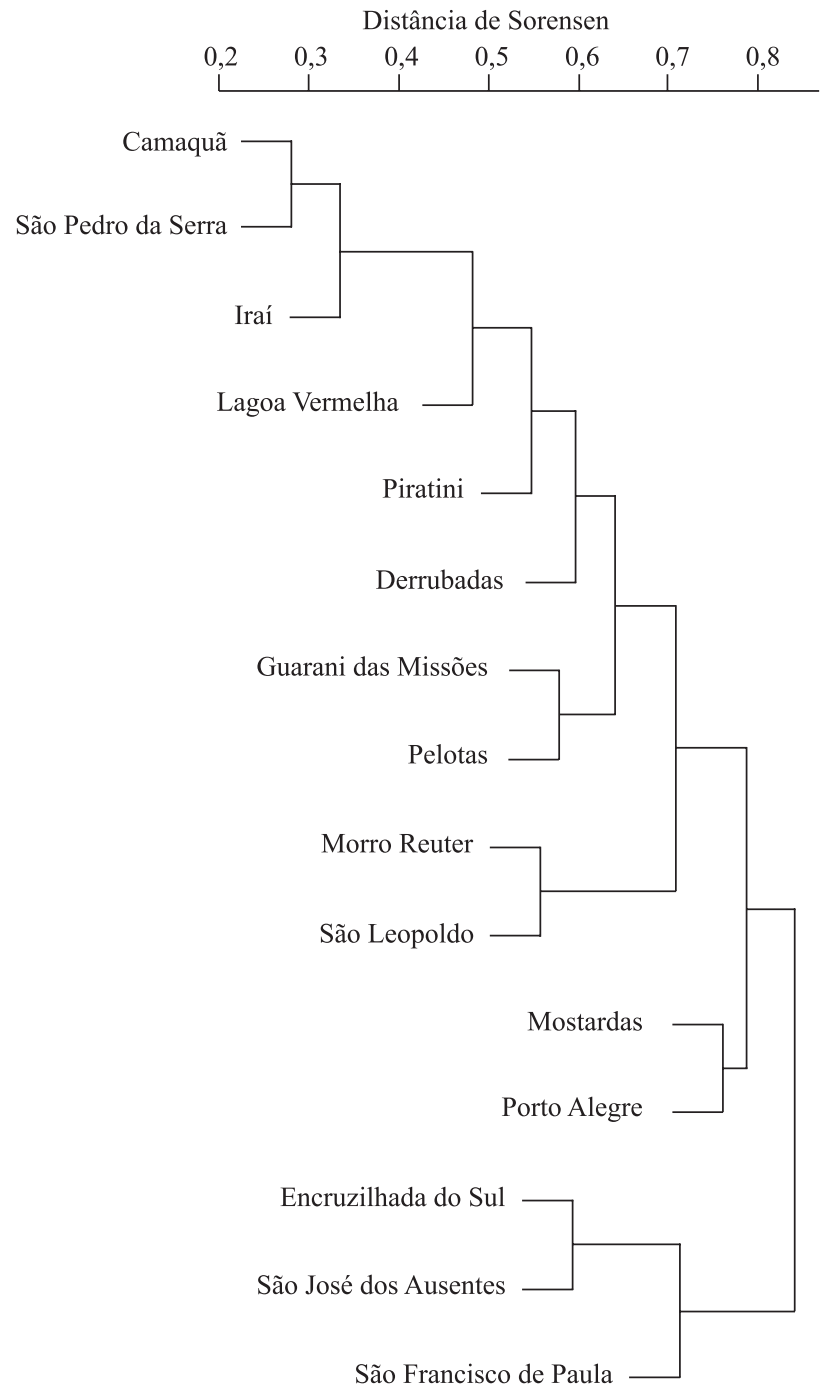

Fig. 2. Dendrograma dos 15 municípios com registro de ocorrência para mais de 20 espécies, utilizando UPGMA como método de ligação e o índice de Sorensen como medida de distância.

distância geográfica (em km) entre as localidades. Todas as análises foram realizadas com o programa $\mathrm{R}$ ( $\mathrm{R}$ Development Core Team 2008).

\section{RESULTADOS}

Ao todo, houve registro de arctiídeos em 55 localidades do RS (Fig. 1, Tab. I). Foram listadas 329 espécies das duas subfamílias que ocorrem na Região Neotropical (Arctiinae e Lithosiinae), das quais $52(15,8 \%)$ foram novos registros para o Estado. A subfamília Arctiinae apresentou o maior número de espécies (91\%, Apêndice I). Foram registrados representantes de nove tribos: Arctiini, Callimorphini, Ctenuchini, Euchromiini, Pericopini e Phaegopterini (Arctiinae), e Cisthenini, Eudesmiini e Lithosiini (Lithosiinae). A riqueza variou de 1 a 154 espécies entre as localidades (Tab. I). Agrupando os dados por bioma, a riqueza total em áreas de
Mata Atlântica foi de 316 espécies e de 50 espécies em áreas de Campos Sulinos.

Nenhuma espécie ocorreu em todas as localidades. Aproximadamente, $33 \%(n=112)$ das espécies foram encontradas em apenas uma localidade e $19 \%(n=64)$ em duas localidades (Apêndice I). As espécies mais comumente encontradas foram: Idalus agastus Dyar, 1910 e Philoros affinis (Rothschild, 1912) (presentes em 15 localidades), Baritius acuminata (Walker, 1856), Cosmosoma centrale (Walker, 1854), Dysschema hilarina (Weymer, 1914), Dysschema sacrifica (Hübner, 1831) e Phaloe cruenta (Hübner, 1823) (presentes em 13 localidades), todas da subfamília Arctiinae (Apêndice I).

Não foi encontrada correlação significativa entre a diversidade beta e a distância geográfica entre as localidades

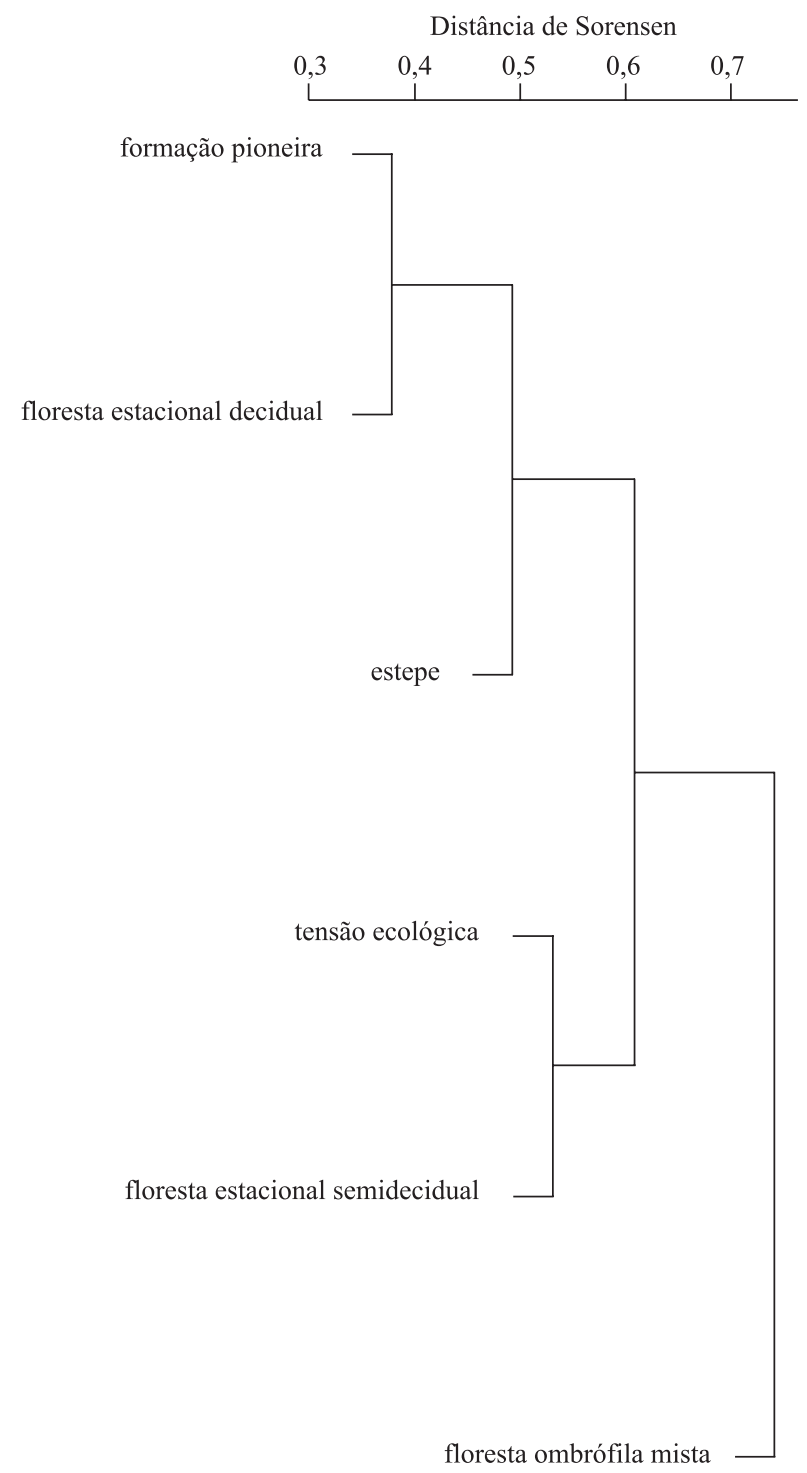

Fig. 3. Dendrograma dos seis tipos de vegetação que ocorrem no RS segundo o IBGE (1993), utilizando UPGMA como método de ligação e o índice de Sorensen como medida de distância. 
Tabela I. Riqueza de espécies de Arctiidae, coordenadas geográficas (latitude sul; longitude oeste), altitude e tipo de vegetação em 55 municípios gaúchos. M= Mata Atlântica e C= Campos Sulinos.

\begin{tabular}{|c|c|c|c|c|c|}
\hline Código & Município & $\begin{array}{l}\text { Altitude } \\
(\mathrm{m})\end{array}$ & $\begin{array}{l}\text { Coordenadas geográficas } \\
\text { (graus decimais) }\end{array}$ & $\begin{array}{l}\text { Tipo de vegetação } \\
\text { (Bioma)* }\end{array}$ & $\begin{array}{l}\text { Riqueza de } \\
\text { espécies }\end{array}$ \\
\hline 1 & Alegrete & 102 & 29,$7831 ; 55,7919$ & estepe $(C)$ & 16 \\
\hline 2 & Bagé & 212 & 31,$3314 ; 54,1066$ & estepe $(\mathrm{C})$ & 2 \\
\hline 3 & Barracão & 764 & 27,$6717 ; 51,4606$ & floresta ombrófila mista (M) & 16 \\
\hline 4 & Bom Jesus & 1046 & 28,$6678 ; 50,4167$ & estepe $(\mathrm{M})$ & 4 \\
\hline 5 & Cachoeira do Sul & 68 & 30,$0392 ; 52,8939$ & área de tensão ecológica (M) & 6 \\
\hline 6 & Camaquã & 144 & 30,$8511 ; 51,8122$ & área de formação pioneira (M) & 103 \\
\hline 7 & Cambará do Sul & 1031 & 29,$0478 ; 50,1447$ & floresta ombrófila densa (M) & 3 \\
\hline 8 & Canela & 837 & 29,$3656 ; 50,8156$ & floresta ombrófila mista (M) & 2 \\
\hline 9 & Canguçu & 386 & 31,$395 ; 52,6756$ & área de formação pioneira $(\mathrm{C})$ & 1 \\
\hline 10 & Capão do Leão & 21 & 31,$7633 ; 52,4839$ & área de formação pioneira (M) & 1 \\
\hline 11 & Cruz Alta & 452 & 28,$6386 ; 53,6064$ & floresta estacional decidual (M) & 1 \\
\hline 12 & Derrubadas & 485 & 27,$2647 ; 53,8608$ & floresta estacional decidual (M) & 78 \\
\hline 13 & Dois Irmãos & 166 & 29,$5803 ; 51,0853$ & área de tensão ecológica $(\mathrm{M})$ & 1 \\
\hline 14 & Dom Pedrito & 141 & 30,$9828 ; 54,6731$ & estepe $(C)$ & 1 \\
\hline 15 & Encruzilhada do Sul & 432 & 30,$5439 ; 52,5219$ & estepe $(C)$ & 22 \\
\hline 16 & Erechim & 783 & 27,$6342 ; 52,2739$ & floresta ombrófila mista (M) & 1 \\
\hline 17 & Estância Velha & 44 & 29,$6483 ; 51,1739$ & área de tensão ecológica (M) & 1 \\
\hline 18 & Estrela & 39 & 29,$5019 ; 51,9664$ & floresta estacional decidual (M) & 1 \\
\hline 19 & Feliz & 68 & 29,$4508 ; 51,3064$ & floresta estacional decidual (M) & 1 \\
\hline 20 & Garibaldi & 617 & 29,$2561 ; 51,5336$ & floresta estacional decidual (M) & 1 \\
\hline 21 & Gramado & 825 & 29,$3786 ; 50,8739$ & floresta ombrófila mista (M) & 1 \\
\hline 22 & Guarani das Missões & 267 & 28,$1408 ; 54,5581$ & floresta estacional decidual (M) & 63 \\
\hline 23 & Ijuí & 328 & 28,$3878 ; 53,9147$ & floresta estacional decidual (M) & 4 \\
\hline 24 & Iraí & 235 & 27,$1936 ; 53,2506$ & floresta estacional decidual (M) & 104 \\
\hline 25 & Ivoti & 127 & $29,591151,1606$ & floresta estacional semidecidual (M) & 11 \\
\hline 26 & Jaquirana & 927 & 28,$8847 ; 50,3578$ & estepe $(\mathrm{M})$ & 1 \\
\hline 27 & Lagoa Vermelha & 830 & 28,$2086 ; 51,5258$ & estepe $(\mathrm{M})$ & 64 \\
\hline 28 & Lomba Grande & 30 & 29,$7500 ; 51,0167$ & área de tensão ecológica (M) & 3 \\
\hline 29 & Maquiné & 12 & 29,$675 ; 50,2072$ & floresta ombrófila densa $(\mathrm{M})$ & 6 \\
\hline 30 & Montenegro & 31 & 29,$6886 ; 51,4611$ & floresta estacional semidecidual (M) & 2 \\
\hline 31 & Morro Reuter & 492 & 29,$5381 ; 51,0808$ & floresta estacional semidecidual (M) & 112 \\
\hline 32 & Mostardas & 17 & 31,$1069 ; 50,9211$ & área de formação pioneira $(\mathrm{M})$ & 28 \\
\hline 33 & Pelotas & 17 & 31,$7719 ; 52,3425$ & área de formação pioneira (M) & 154 \\
\hline 34 & Piratini & 225 & 31,$4481 ; 53,1042$ & estepe $(\mathrm{C})$ & 51 \\
\hline 35 & Porto Alegre & 3 & 30,$0331 ; 51,2300$ & área de tensão ecológica (M) & 30 \\
\hline 36 & Rio Grande & 5 & 32,$035 ; 52,0986$ & área de formação pioneira (M) & 5 \\
\hline 37 & Rosário do Sul & 125 & 30,$2583 ; 54,9142$ & estepe $(C)$ & 2 \\
\hline 38 & Salvador do Sul & 493 & 29,$4383 ; 51,5114$ & área de tensão ecológica (M) & 3 \\
\hline 39 & Santa Maria & 151 & 29,$6842 ; 53,8069$ & área de tensão ecológica (M) & 18 \\
\hline 40 & Santa Rosa & 277 & 27,$8708 ; 54,4814$ & floresta estacional decidual (M) & 1 \\
\hline 41 & Santana do Livramento & 208 & 30,$8908 ; 55,5328$ & estepe $(C)$ & 5 \\
\hline 42 & São Borja & 123 & 28,$6606 ; 56,0044$ & estepe (C) & 1 \\
\hline 43 & São Francisco de Paula & 907 & 29,$4481 ; 50,5836$ & floresta ombrófila mista (M) & 52 \\
\hline 44 & São Jerônimo & 29 & 29,$9592 ; 51,7222$ & estepe $(\mathrm{C})$ & 2 \\
\hline 45 & São José do Hortêncio & 100 & 29,$5306 ; 51,2481$ & floresta estacional semidecidual (M) & 1 \\
\hline 46 & São José dos Ausentes & 1116 & 28,$7483 ; 50,0658$ & floresta ombrófila mista $(\mathrm{M})$ & 27 \\
\hline 47 & São Leopoldo & 15 & 29,$7603 ; 51,1472$ & área de tensão ecológica (M) & 42 \\
\hline 48 & São Lourenço do Sul & 19 & 31,$3653 ; 51,9783$ & área de formação pioneira $(\mathrm{C})$ & 2 \\
\hline 49 & São Luiz Gonzaga & 231 & 28,$4083 ; 54,9608$ & estepe $(C)$ & 17 \\
\hline 50 & São Pedro da Serra & 556 & 29,$4211 ; 51,5133$ & floresta estacional decidual (M) & 98 \\
\hline 51 & Sarandi & 503 & 27,$9439 ; 52,9231$ & floresta estacional decidual (M) & 10 \\
\hline 52 & Torres & 16 & 29,$3353 ; 49,7269$ & floresta ombrófila densa (M) & 1 \\
\hline 53 & Tramandaí & 8 & 29,$9847 ; 50,1336$ & área de formação pioneira (M) & 1 \\
\hline 54 & Vacaria & 971 & 28,$5122 ; 50,9339$ & estepe $(\mathrm{M})$ & 2 \\
\hline 55 & Vila Maria & 455 & 28,$5347 ; 52,1536$ & floresta ombrófila mista (M) & 16 \\
\hline
\end{tabular}

* Classificação segundo o Mapa de Vegetal do Brasil (IBGE 2004). 
$\left(r^{2}=0,003 ; p=0,45\right)$. A análise de UPGMA para a fauna coletada nas diferentes localidades do RS (Fig. 2) gerou dois grandes grupos: um formado por Encruzilhada do Sul, São José dos Ausentes e São Francisco de Paula e outro pelas demais localidades. Já a análise de UPGMA para a fauna coletada em diferentes tipos de vegetação (Fig. 3) mostrou que a fauna de Arctiidae em áreas de Floresta Ombrófila Mista apresenta uma composição bem diferente da fauna dos demais tipos de vegetação. Na segunda divisão, a fauna da Floresta Estacional Semidecidual e a da Área de Tensão Ecológica formaram um grupo e os arctiídeos encontrados em regiões de vegetação de Floresta Estacional Decidual, área de formação pioneira (restingas e banhados) e estepe formaram um outro grupo.

\section{DISCUSSÃO}

As espécies de Arctiidae registradas neste estudo correspondem a 5,6\% da fauna Neotropical $(n=5931$, Heppner 1991) e a $16,5 \%$ da fauna estimada para o Brasil $(n=2000$, Brown Jr \& Freitas 1999). Teston \& Corseuil (2002, 2003a, 2003b, 2004), baseados em dados de coleções e de coletas, registraram 327 espécies de Arctiidae para o RS. Com o acréscimo dos 52 novos registros obtidos em nosso estudo, a riqueza total de Arctiidae no RS passa a ser de 379.

Marinoni \& Dutra (1996) amostraram 92 espécies das tribos Ctenuchini e Euchromiini em oito localidades no Estado do Paraná (PR). No RS, foram registradas 130 espécies dessas tribos, sendo 42 (32\%) comuns à fauna do PR. Quatro espécies dessas tribos, Aclytia heber (Cramer, 1780), P. affinis, Aristodaema hanga (Herrich-Schäffer, 1854) e C. centrale, foram registradas em um grande número de localidades no RS. Essas espécies parecem ter uma distribuição ampla no sul do Brasil, já que também foram encontradas em quase todas as localidades amostradas por Marinoni \& Dutra (1996) no PR e por Teston \& Corseuil (2004) no RS. Essas espécies também foram coletadas nas regiões sudeste (estado de São Paulo) e centro-oeste (estados de Goiás e Tocantins) do país (Ferro \& Diniz 2007a, 2007b).

As riquezas de Arctiidae foram maiores em locais com vegetação florestal (pertencentes ao bioma da Mata Attântica) do que em locais com vegetação campestre (bioma Campos Sulinos). Teston \& Corseuil (2004) e Teston et al. (2006) também observaram riquezas de espécies superiores dessas mariposas em sítios de Mata Atlântica do que de Campos Sulinos. Do mesmo modo, Specht et al. (2005) e Cook \& Graham (1996) verificaram que os ambientes florestais apresentaram os maiores índices de diversidade para Noctuidae e mariposas de regiões temperadas, respectivamente. Ferro \& Diniz (2007b) também verificaram um elevado valor de riqueza de Arctiidae (237 espécies) num sítio de Mata Atlântica do Estado de São Paulo e Hilt \& Fiedler (2006) amostraram 287 espécies de Arctiidae numa floresta úmida do Equador. Entretanto, esses dados devem ser vistos com cautela, pois a fauna das áreas de vegetação campestre é pouco estudada. No RS, existem registros de arctí́deos em apenas 12 sítios do bioma Campos Sulinos e na Mata Atlântica são 43 sítios.
O decréscimo da similaridade com a distância tem sido observado em vários táxons vegetais (e.g. Qian et al. 2005) e animais (e. g. Steinitz et al. 2006). Entretanto, nossas análises mostraram a inexistência de correlação entre a similaridade de Arctiidae e a distância geográfica entre as localidades. Isso sugere que a configuração espacial do ambiente (tamanho e isolamento de hábitats e características da matriz que circunda o hábitat) não influencia de forma significativa a locomoção destas mariposas entre as paisagens. De fato, grande parte das espécies de arctiídeos registradas para o RS possui grande capacidade de dispersão (Hilt 2005). Além disso, a maioria das larvas dessas mariposas são polífagas (Hilt \& Fiedler 2006). O amplo espectro de plantas hospedeiras facilita o sucesso no estabelecimento de uma população em um novo hábitat, pois a chance da mariposa encontrar uma fonte alimentar nesse novo hábitat é maior. Contudo, Ferro \& Diniz (2007a) encontraram uma forte correlação entre a distância geográfica entre as localidades e a similaridade da fauna de Arctiidae no Cerrado. Uma possível explicação para esse resultado contrário poderia ser os diferentes tipos de dados usados nesses estudos. Nosso trabalho utilizou dados de coleções e as autoras utilizaram dados de coletas padronizadas. Sabe-se que os exemplares de coleções são provenientes de coletas realizadas por vários pesquisadores, predominantemente taxonomistas interessados em certos grupos, que utilizavam diferentes métodos, períodos e esforços de amostragens. Essa falta de padronização das coletas pode ter aumentado a variabilidade de nossos dados, dificultando a observação de uma correlação significativa.

A literatura apresenta vários estudos que demonstram que as características da vegetação influenciam a riqueza e composição de Lepidoptera (e. g. Brown Jr \& Gifford 2002). A análise de UPGMA para os seis principais tipos de vegetação do RS indicou que a fauna de Arctiidae responde às mudanças na flora. Estudos realizados no PR (Marinoni \& Dutra 1996), no Cerrado brasileiro (Ferro \& Diniz 2007a) e na Austrália (Kitching et al. 2000) também observaram uma relação entre a composição de Arctiinae e o tipo de vegetação. A partir desse resultado, pode-se sugerir que as diferenças entre os tipos de vegetação explicam o padrão de diversidade beta encontrado para os Arctiidae do RS. Sabe-se, contudo, que a riqueza e a composição de espécies de Lepidoptera estão relacionadas com uma série de outros fatores, principalmente o clima, altitude e solo (Brown Jr \& Gifford 2002). A relação da composição da fauna dessas mariposas com essas outras variáveis ambientais será objeto de estudos futuros.

Agradecimentos. Aos curadores das coleções visitadas, Olaf H. H. Mielke, Marcelo Duarte, Jane Costa, Alexandre Soares, Eduardo J. E. e Silva, Mirtes Mello, Élvia E. S. Vianna, Luciano de A. Moura, Vera Wolf e Fernando Z. da Cruz, por permitir o acesso às coleções entomológicas, pelo apoio logístico e pela atenção dispensada. A Vitor O. Becker pelo empréstimo de bibliografia, pelo acesso à sua coleção, pela permissão do registro fotográfico e pelo auxílio nas identificações das espécies de Arctiidae. A Alfred Moser, pelo empréstimo de bibliografia. VGF recebeu financiamento do CNPq (processo 141715/ 2005-7). 


\section{REFERÊNCIAS}

Biezanko, C. M. 1983. Ctenuchidae, Nolidae, Arctiidae e Pericopidae da Zona Sudeste do Rio Grande do Sul. Revista do Centro de Ciências Rurais 13: 229-263.

Biezanko, C. M. 1985. Ctenuchidae, Arctiidae e Pericopidae da Zona Missioneira do Rio Grande do Sul. Revista do Centro de Ciências Rurais 15: 189-210.

Blackburn, T. M. \& K. J. Gaston. 1996. The distribution of bird species in the New World: patterns in species turnover. Oikos 77: 146152 .

Bridgewater, S.; J. A. Ratter \& J. F. Ribeiro. 2004. Biogeographic patterns, B-diversity and dominance in the cerrado biome of Brazil. Biodiversity and Conservation 13: 2295-2318.

Brown Jr, K. S. \& A. V. L. Freitas. 1999. Lepidoptera, p. 225-243. In: C. R. F. Brandão \& E. M. Cancello (eds.). Biodiversidade do Estado de São Paulo: síntese do conhecimento ao final do século XX. Volume 5. Invertebrados terrestres. São Paulo, Fapesp, 279 p

Brown Jr, K. S. \& D. R Gifford. 2002. Lepidoptera in the cerrado landscape and the conservation of vegetation, soil and topographical mosaics, p. 201-217. In: P. S. Oliveira \& R. J. Marquis (eds.). The Cerrados of Brazil: Ecology and Natural History of a Neotropical Savanna. New York, Columbia University Press, $398 \mathrm{p}$.

Cook, L. M. \& C. S. Graham. 1996. Eveness and species number in some moth populations. Biological Journal of the Linnean Society 58: 75-84.

Ferro, V. G. \& I. R. Diniz. 2007a. Composição de espécies de Arctiidae (Insecta, Lepidoptera) em áreas de Cerrado. Revista Brasileira de Zoologia 24: 635-646.

Ferro, V. G. \& I. R. Diniz. 2007b. Arctiidae (Insecta: Lepidoptera) da Estação Biológica de Boracéia (Salesópolis, SP, Brasil). Biota Neotropica 7. http://www.biotaneotropica.org.br/v7n3/pt/ fullpaper?bn03107032007+pt. Acesso em novembro de 2008.

Heppner, J. B. 1991. Faunal regions and the diversity of Lepidoptera. Tropical Lepidoptera 2: 1-85.

Hilt, N. 2005. Diversity and species composition of two different moth families (Lepidoptera: Arctiidae vs. Geometridae) along a successional gradient in the Ecuadorian Andes. Tese de doutorado, Universidade de Bayreuth, 251 p. http://opus.ub.unibayreuth.de/volltexte/2006/201/pdf/DISS_HILT.pdf. Acesso em novembro de 2008 .

Hilt, N. \& K. Fiedler. 2006. Arctiidae moth ensembles along a successional gradient in the Ecuadorian montane rain forest zone: how different are subfamilies and tribes? Journal of Biogeography 33: $108-120$.

Hilty, J. \& A. Merenlender. 2000. Faunal indicator taxa selection for monitoring ecossystem health. Biological Conservation 92 : 185-197.
IBGE. 2004. Mapa de Vegetação do Brasil. ftp://geoftp.ibge.gov.br/ mapas/tematicos/ mapas_murais/ vegetacao.pdf. Acesso em novembro de 2008.

Kitching, R. L.; A. G. Orr; L. Thalib; H. Mitchell; M. S. Hopkins \& A. W. Graham. 2000. Moths assemblages as indicators of environmental quality in remnants of upland Australian rain forest. Journal of Applied Ecology 37: 284-297.

Legendre, P. 1993. Spatial autocorrelation: trouble or new paradigm? Ecology 74: 1659-1673.

Marinoni, R. C. \& R. R. C. Dutra. 1996. Levantamento da fauna entomológica do Estado do Paraná. II. Ctenuchidae (Lepidoptera). Revista Brasileira de Zoologia 13: 435-461.

Nekola, J. C. \& P. S. White. 1999. The distance decay of similarity in biogeography and ecology. Journal of Biogeography 26: 867878.

Qian, H.; R. E. Ricklefs \& P. S. White. 2005. Beta diversity of angiosperms in temperate floras of eastern Asia and eastern North America. Ecology Letters 8: 15-22.

R Development Core Team. 2008. R: A language and environment for statistical computing. Version 2.7.1. User's guide and aplication published: http://www.R-project.org. Acesso em novembro de 2008.

Romanowski, H. P. \& G. Buss. 1997. Biodiversidade e conservação, p. 61-85. In: A. Escosteguy (ed.). Queridos Animais. Porto Alegre, L \& PM Editores, 204p.

Specht, A.; J. A. Teston; R. A. Di Mare \& E. Corseuil. 2005. Noctuídeos (Lepidoptera, Noctuidae) coletados em quatro áreas estaduais de conservação do Rio Grande do Sul. Revista Brasileira de Entomologia 49: 130-140.

Steinitz, O.; J. Heller; A. Tsoar; D. Rotem \& R. Kadmon. 2006. Environment, dispersal and patterns of species similarity. Journal of Biogeography 33: 1044-1054.

Teston, J. A. \& E. Corseuil. 2002. Arctiinae (Lepidoptera, Arctiidae) ocorrentes no Rio Grande do Sul, Brasil. Parte I. Pericopini. Biociências 10: 261-268.

Teston, J. A. \& E. Corseuil. 2003a. Arctiinae (Lepidoptera, Arctiidae) ocorrentes no Rio Grande do Sul, Brasil. Parte II. Arctiini, Callimorphini e Phaegopterini. Biociências 11: 69-80.

Teston, J. A. \& E. Corseuil. 2003b. Arctiinae (Lepidoptera, Arctiidae) ocorrentes no Rio Grande do Sul, Brasil. Parte III. Ctenuchini e Euchromiini. Biociências 11: 81-90.

Teston, J. A. \& E. Corseuil. 2004. Diversidade de Arctiinae (Lepidoptera, Arctiidae) capturados com armadilha luminosa, em seis comunidades no Rio Grande do Sul, Brasil. Revista Brasileira de Entomologia 48: 77-90.

Teston, J. A.; A. Specht; R. A. Di Mare \& E. Corseuil. 2006. Arctiinae (Lepidoptera, Arctiidae) coletados em unidades de conservação estaduais do Rio Grande do Sul, Brasil. Revista Brasileira de Entomologia 50: 280-286.

Whittaker, R. H. 1972. Evolution and measurement of species diversity. Taxon 21: 213-251. 
Apêndice I. Arctiídeos com registro de ocorrência para o Rio Grande do Sul. Asteriscos indicam novos registros para o Estado.

\begin{tabular}{ll}
\hline Espécies & $\begin{array}{l}\text { Localidades } \\
\text { (ver Tabela I) }\end{array}$ \\
\hline
\end{tabular}

Subfamília Arctiinae (302 espécies)

Tribo Arctiini (28 espécies)

Hypercompe abdominalis (Walker, [1865])* 29, 31, 33

Hypercompe brasiliensis (Oberthür, 1881) 6, 12, 31, 50

Hypercompe cunigunda (Stoll, 1781)

Hypercompe detecta (Oberthür, 1881)

Hypercompe heterogena (Oberthür, 1881)

Hypercompe indecisa (Walker, 1855)

Hypercompe kinkelini (Burmeister, 1878)

Hypercompe orsa (Cramer, 1777)

Isia alcumena (Berg, 1882) *

Isia intricata Walker, 1855

Paracles albescens (Hampson, 1901)

Paracles aurantiaca (Rothschild, 1910) *

Paracles azollae (Berg, 1877)

Paracles bilinea (Schaus, 1901)

Paracles burmeisteri (Berg, 1877) *

Paracles cajetani (Rothschild, 1910) *

Paracles costata (Burmeister, 1878)

Paracles fervida (Schaus, 1901)

Paracles fusca (Walker, 1856)

Paracles pallidivena (Schaus, 1904)

Paracles paula (Schaus, 1896)

Paracles persimilis (Burmeister, 1878)

Paracles phaeocera (Hampson, 1905)

Paracles uniformis (Jones, 1912) *

Paracles variegata (Schaus, 1896)

Paracles vulpina (Hübner, [1825])

Virbia divisa (Walker, 1864)

Virbia ovata Rothschild, 1910 *

Tribo Callimorphini (1 espécie)

Utetheisa ornatrix (Linnaeus, 1758)

Tribo Ctenuchini (70 espécies)

Aclytia heber (Cramer, 1780)

Aclytia jonesi Rothschild, 1912

Aclytia terra Schaus, 1896

Aethria melanobasis (Druce, 1897)

Aethria ornata (Ménétriés, 1857) *

Agyrta albisparsa Hampson, 1898

Antichloris eriphia (Fabricius, 1776)

Aphra flavicosta (Herrich-Schäffer, 1855)

Argyroeides braco (Herrich-Schäffer, 1855)

Argyroeides flaviceps Hampson, 1898

Argyroeides sanguinea Schaus, 1896

Argyroeides vespina Schaus, 1901

Callopepla grandis Rothschild, 1912

Callopepla inachia (Schaus, 1892)

Callopepla similis (Heylaerts, 1890)

Centronia melanitis Hübner, 1827

Correbia lycoides (Walker, 1854)

Ctenucha divisa Walker, 1856

Ctenucha jonesi Rothschild, 1912

Ctenucha mortia Schaus, 1901

Ctenucha vittigera (Blanchard, 1852)

Cyanopepla jucunda (Walker, 1854)

Cyanopepla julia Druce, 1883

Cyanopepla orbona (Druce, 1893)
$24,34,50,55$

$6,24,32,34,43,50$

6,55

$33,34,35$

$15,31,32,33,34,36,39$, 55

39

46

$8,12,24,27,31,39,50$

6,27

33

$32,33,34,35,36$

$6,15,24,27,31,33,34$, 46,50

33

33

6, 33, 34

33

$6,12,22,23,27,33,34$,

39,50

6,33

$6,33,39$

33

33

33

$3,6,15,27,31,33,34$,

$43,46,51$

$5,22,32,33$

31

33

$1,6,22,24,27,33,38$, 43,47

$1,6,12,24,27,31,33$, $34,37,47,50,55$

$24,33,55$

$1,6,12,22,24,27,32$, $33,34,41,50$

33,45

33

6,47

35

39

$22,24,25$

$22,24,33$

$2,12,15,22,24,25,33$

$5,24,33$

$33,34,35$

$12,22,31,33,43,50$

22,33

22

$31,33,43$

$22,24,26,27,28,54$

$22,25,30,31,33,47,49$

31,33

$7,25,31,43,46,47$

$2,3,6,7,22,25,31,33$,

$43,46,55$

33

55
Apêndice I. Continuação.

Espécies Localidades

Delphyre brunnea (Druce, 1898)

Delphyre hebes Walker, 1854

Delphyre pyroperas Hampson, 1911

Delphyre roseiceps Dognin, 1909

Dinia eagrus (Cramer, 1779)

Diptilon aterea Schaus, 1901

Diptilon doeri (Schaus, 1892)

Diptilon halterata (Fabricius, 1775)

Diptilon telamonaphorum Prittwitz, 1870

Episcepsis endodasia Hampson, 1898

Episcepsis obsoleta (Burmeister, 1878)

Eucereon apicalis (Walker, 1856)

Eucereon arenosum Butler, 1877

Eucereon capsica (Schaus, 1896)

Eucereon chalcodon Druce, 1893

Eucereon dilutius Rothschild, 1912

Eucereon discolor (Walker, 1856)

Eucereon ladas Schaus, 1892

Eucereon latifascia Walker, 1856 *

Eucereon marcata Schaus, 1901

Eucereon nubilosa Rothschild, 1912 *

Eucereon pallescens Rothschild, 1912 *

Eucereon quadricolor (Walker, 1855)

Eucereon rosa (Walker, 1854)

Eucereon striatum (Druce, 1889)

Eucereon tarona Hampson, 1898 *

Eucereon vestalis Schaus, 1892

Euceriodes pallada (Druce, 1906)

Euclera rubricincta (Burmeister, 1878)

Galethalea pica (Walker, 1855)

Hyaleucerea mundula (Berg, 1882)

Napata castra Hampson, 1898

Nelphe confinis (Herrich-Schäffer, [1855])

Nelphe mara (Sepp, 1848)

(ver Tabela I)

12,49

33

$12,22,24,31,47,50$

$6,24,32,50$

$17,24,31,47$

35,50

24

$12,24,49$

$12,22,24,33$

$3,6,12,24,50$

33

31,50

6,50

24,50

$6,22,31,32,33,47,50$

33

33,50

$3,6,24,27,31,33,43$,

51

$12,31,47$

24,50

31,33

32,33

$6,24,31,33,35,39,50$

$6,12,24,31,33,34,47$,

49,50

$6,31,33,50$

33

$22,31,33$

$6,12,24,27,33,50$

$33,34,41,55$

$6,31,43,50$

50

$3,4,5,7,35,43,46$

33

$6,24,27,50$

$12,22,25,31,33,47,51$

Paraethria triseriata (Herrich-Schäffer, 1855)

Philoros affinis (Rothschild, 1912)

$1,6,22,24,27,31,32,33$,

$34,37,41,46,47,49,50$

Pseudohyaleucerea vulnerata (Butler, 1875) 6, 12, 22, 24, 27, 31, 33, 50

Pseudosphex noverca Schaus, 1901

Pseudosphex polybia Kaye, 1911

Pseudosphex polybioides Burmeister, 1878

Sciopsyche tropica (Walker, 1854)

Theages leucophaea (Walker, 1855)

Tipulodes ima Boisduval, 1833

$24,50,55$

33,53

33

24

$22,31,33,43,50,51$

$1,6,12,24,27,31,33$, $35,39,50$

33,48

33,39

Trichura cerberus (Pallas, 1772)
Trichura coarctata (Drury,1773)

Tribo Euchromiini (60 espécies)

Aristodaema hanga (Herrich-Schäffer, [1854]) 6, 15, 22, 24, 27, 31, 33,

Cosmosoma auge (Linnaeus, 1767)

$43,46,49,50,51$

$6,22,24,31,33,46$,

47,50

$6,12,15,24,27,31,32$, $33,34,35,47,50,51$ 31

Cosmosoma elegans Butler, 1876

Cosmosoma leuconotum Hampson, 1898

Cosmosoma pellucida Lathy, 1899 *

Cosmosoma plutona Schaus, 1894

Cosmosoma telephus (Walker, 1854)

Cosmosoma teuthras (Walker, 1854)

Cosmosoma xanthistis Hampson, 1898 *

Dixophlebia holophaea Hampson, 1909 *

Dycladia lucetius (Stoll, 1781)
$6,12,22,24,27,39,49$

22

24

24,50

24,49

47

31

$1,6,12,24,27,31,33$ $34,35,47,50$ 
Apêndice I. Continuação.

\begin{tabular}{|c|c|c|c|}
\hline Espécies & $\begin{array}{l}\text { Localidades } \\
\text { (ver Tabela I) }\end{array}$ & Espécies & $\begin{array}{l}\text { Localidades } \\
\text { (ver Tabela I) }\end{array}$ \\
\hline Eurota helena (Herrich-Schäffer, 1854) & $\begin{array}{l}1,6,22,24,27,33,34, \\
35,50\end{array}$ & $\begin{array}{l}\text { Dysschema irene (Druce, 1885) } \\
\text { Dysschema lucifer (Butler, 1873) }\end{array}$ & $\begin{array}{l}12,13,39 \\
31\end{array}$ \\
\hline Eurota hermione (Burmeister, 1878) & 22,33 & Dysschema luctuosa (Dognin, 1919) & $3,31,43,46$ \\
\hline Eurota herricki Butler, 1876 & $\begin{array}{l}1,6,22,27,33,34,35 \\
48,50\end{array}$ & $\begin{array}{l}\text { Dysschema neda (Klug, 1836) } \\
\text { Dysschema picta (Guérin-Méneville, [1844]) }\end{array}$ & $\begin{array}{l}3,6,8,11,27,31,35,50 \\
6,12,24,50\end{array}$ \\
\hline Eurota histrio (Guérin, 1843) & 33 & Dysschema sacrifica (Hübner, [1831]) & $6,10,12,24,27,31,32$, \\
\hline Eurota joergenseni Orfila, 1931 & 22,33 & & $33,34,35,38,50,51$ \\
\hline Eurota nigricincta Hampson, 1907 & 22 & Dysschema trapeziata (Walker, [1865]) & $15,20,27,43,46$ \\
\hline Eurota picta (Herrich-Schäffer, 1853) & $22,39,47$ & Dysschema tricolor (Sulzer, 1776) & 29 \\
\hline Eurota schausi Hampson, 1898 & 3 & Euchlaenidia transcisa (Walker, 1854) & $6,24,31,34,35,39,47,50$ \\
\hline Eurota stictibasis Hampson, 1898 & 27 & Hyalurga syma (Walker, 1854) & 24,35 \\
\hline Eurota strigiventris (Guérin, 1843) & $22,33,35$ & Hypocrita bicolora (Sulzer, 1776) & $12,24,25,28,33,36,47$ \\
\hline Gymnelia xanthogastra (Perty, 1834) & 12,40 & Phaloe cruenta (Hübner, 1823) & $6,12,22,24,25,29,31$, \\
\hline Holophaea erharda Schaus, 1927 & $5,6,24,33,34,50$ & & $33,34,35,47,49,50$ \\
\hline Homoeocera acuminata (Walker, 1856) & 3 & Tribo Phaegopterini (123 espécies) & \\
\hline Horama flavata Jones, $1908 *$ & 22,33 & Aemilia asignata Hampson, 1901 & $6,24,50$ \\
\hline Horama panthalon (Fabricius, 1793) & $6,22,24$ & Aemilia pagana (Schaus, 1894) & 43 \\
\hline Ichoria chalcomedusa Druce, 1893 & $12,24,50$ & Agaraea semivitrea Rothschild, 1909 & $6,24,31,32,34,47,50,51$ \\
\hline Ichoria tricincta (Herrich-Schäffer, 1855) & $27,31,50$ & Agaraea uniformis (Hampson, 18 & 12,31 \\
\hline Isanthrene melas (Cramer, 1775) * & 14 & Amaxia corata Schaus, 1921 & 6 \\
\hline Isanthrene ustrina Hübner, 1827 & 33 & Amaxia hebe Schaus, 1892 & $6,31,50$ \\
\hline Lepidoneiva erubescens (Butler, 1876) & $24,33,50$ & Ammalo helops (Cramer, 1775) & $22,23,24,31,32$, \\
\hline Loxophlebia brasiliensis (Rothschild, 1911) * & 28,47 & & 33,3 \\
\hline Macrocneme caerulescens Dognin, 1906 * & 33 & Baritius acuminata (Walker, 1856) & $1,6,12,22,24,27,31$, \\
\hline Macrocneme cupreipennis Walker, 1856 & $6,12,24,34,43,50$ & & $33,34,39,46,49,50$ \\
\hline Macrocneme lades (Cramer, 1776) * & $6,24,33$ & Baritius eleutheroides Rothschild, $1909 *$ & 52 \\
\hline Macrocneme leucostigma (Perty, 1833) & $1,12,22,33$ & Bernathonomus piperita (Herrich-Schäffer, & \\
\hline Macrocneme pelotas Dietz, 1994 & 6,33 & $[1855])$ & 31,43 \\
\hline Mallodeta clavata (Walker, 1854) & $6,12,31,50$ & Bertholdia almeidai Travassos, $1950 *$ & 33 \\
\hline Mallodeta sanguipuncta Druce, 1898 & 43,31 & Bertholdia pseudofumida Travassos, $1950 *$ & 12 \\
\hline Mesothen desperata (Walker, 1856) & $6,12,24,33,50$ & Bertholdia soror Dyar, 1901 & $1,6,22,24,27,33,34,50$ \\
\hline Mesothen pyrrhina Jones, $1914 *$ & 33 & Bertholdia specularis (Herrich-Schäffer, & \\
\hline Metamya flavia (Schaus, 1898) & 12 & & $6,24,50$ \\
\hline Methysia aenetus (Schaus, 1896) & 31,47 & Bertholdia steinbachi Rothschild, 1909 & 35 \\
\hline Mirandisca harpalyce (Schaus, 1892) & $22,24,31,43,50$ & Biturix rectilinea (Burmeister, 1878) & $1,6,15,27,31,33,50$ \\
\hline Mystrocneme varipes (Walker, 1854) * & $33-2$ & Carales astur (Cramer, 1777) & $6,12,24,27,33,35,50$ \\
\hline Neotrichura nigripes (Heylaerts, 1890) & $\begin{array}{l}12,15,24,29,31,33,43 \\
46,49,50\end{array}$ & $\begin{array}{l}\text { Carales maculicollis Walker, } 1855 * \\
\text { Cresera ilus (Cramer, 1776) * }\end{array}$ & $\begin{array}{l}12,31,33,47 \\
35\end{array}$ \\
\hline Nyridela chalciope (Hübner, [1831]) & 6 & Demolis albicostata Hampson, 1901 & $1,6,12,22,24,32,33$, \\
\hline Phoenicoprocta analis Schrottky, 1909 & $\begin{array}{l}12,22,24,27,31,33,47 \\
49,50\end{array}$ & 皮 & $\begin{array}{l}34,35 \\
15,31\end{array}$ \\
\hline Phoenicoprocta rubiventer Hampson, 1898 & 34 & 55]) & 6,2 \\
\hline Phoenicoprocta teda (Walker, 1854) & $5,6,22,24,30,33,47$ & $*$ & 46 \\
\hline Phoeniostacta haematobasis Hampson, 1898 & $3,22,33$ & Elysius c & 33 \\
\hline Poliopastea indisticta (Butler, 1876) & $6,22,24,33,50$ & $910 *$ & 31 \\
\hline Pseudomya tipulina (Hübner, 1812) * & $12,31,47$ & , 1856)* & 31 \\
\hline Psilopleura haemasoma (Curtis, 1812) & 3 & 1910 & 3,6 , \\
\hline Psilopleura sanguipuncta Hampson, 1898 & $6,27,41$ & & 46 \\
\hline Ptychotrichos elongata Schaus, 1905 & 43 & 905 & $6,12,22,24,27,31,33,50$ \\
\hline Rezia cardinale (Hampson, 1898) * & 43 & Euchaetes $r$ & $27,31,43$ \\
\hline Rhynchopyga meisteri (Berg, 1883) & $\begin{array}{l}6,22,24,27,31,33,34 \\
43,50\end{array}$ & $\begin{array}{l}\text { Eupseudosoma involuta (Sepp, [1849]) } \\
\text { Graphea marmorea Schaus, } 1894\end{array}$ & $\begin{array}{l}12,24,31,47 \\
12,22\end{array}$ \\
\hline Saurita bipuncta Hampson, 1898 & $6,22,24,33,50$ & Halysidota brasiliensis Rothschild, 1909 & 24,33 \\
\hline Saurita cassandra (Linnaeus, 1758) & $\begin{array}{l}6,19,22,24,31,33,47 \\
49,50\end{array}$ & $\begin{array}{l}\text { Halysidota pearsoni Watson, } 1980 \\
\text { Halysidota striata Jones, } 1908\end{array}$ & $\begin{array}{l}6,24,27,34,50 \\
4,22,27,31,43,46\end{array}$ \\
\hline Tribo Perico & & Hemihyalea diminuta (Walker, 1855) & 6,34 \\
\hline Calodesma collaris (Drury, 1782) & $12,24,33$ & & $22,24,27,31$ \\
\hline Calodesma dioptis (Felder, 1874) & 12,24 & Hyperthaema albipuncta Schaus, 1901 & $3,27,33,34$ \\
\hline Calodesma maculifrons (Walker, 1865) * & 33 & Hyperthaema sanguineata (Walker, [1865]) & 12,24 \\
\hline Dysschema amphissa (Geyer, 1832) & $6,12,15,24,25,31,33,50$ & Hyperthaema signatus (Walker, 1862) & 6,34 \\
\hline Dysschema boisduvallii (van der Hoeven, 1840) & 41 & Hypidalia enervis (Schaus, 1894) & $3,6,15,27,32,33,43$ \\
\hline Dysschema centenaria (Burmeister, 1878) & 33 & & 46,55 \\
\hline Dysschema fantasma (Butler, 1873) & $3,4,43$ & Idalus agastus Dyar, 1910 & $1,5,6,12,15,24,27,31$, \\
\hline Dysschema hilarina (Weymer, 1914) & $\begin{array}{l}6,12,15,27,31,33,34 \\
35,38,43,46,50,51\end{array}$ & hild, 1909) * & $\begin{array}{l}32,33,34,35,46,47,50 \\
33\end{array}$ \\
\hline Dysschema hypoxantha Hübner, 1818 & 12,24 & Idalus citrina Druce, 1890 & 6 \\
\hline
\end{tabular}

Apêndice I. Continuação.

Dysschema irene (Druce, 1885)

Dysschema lucifer (Butler, 1873)

Dysschema neda (Klug, 1836)

Dysschema picta (Guérin-Méneville, [1844])

Dysschema trapeziata (Walker, [1865])

Dysschema tricolor (Sulzer, 1776)

Hyalurga syma (Walker, 1854)

Hypocrita bicolora (Sulzer, 1776)

Tribo Phaegopterini (123 espécies)

Agaraea semivitrea Rothschild, 1909

mpson, 1898)

ia corata Schaus, 1921

Axia hebe Schaus, 1892

Baritius acuminata (Walker, 1856)

Baritius eleutheroides Rothschild, 1909 *

assos, 1950 [1853])

Carales maculicollis Walker, 1855 *

Cresera ilus (Cramer, 1776) *

Demolis flavithorax Rothschild, 1909 *

Echeta divisa (Herrich-Schäffer, [1855])

Echeta minerva (Schaus, 1905)

Elysius discoplaga (Walker, 1856) *

Eupseudosoma involuta (Sepp, [1849])

Graphea marmorea Schaus, 1894

Hyalarctia sericea Schaus, 1901 
Apêndice I. Continuação.

\begin{tabular}{|c|c|}
\hline Espécies & $\begin{array}{l}\text { Localidades } \\
\text { (ver Tabela I) }\end{array}$ \\
\hline Idalus critheis Druce, 1884 & 33,35 \\
\hline Idalus noiva (Jones, 1914) & 6,43 \\
\hline Idalus vitrea (Cramer, 1780) & 24 \\
\hline Ischnocampa lugubris (Schaus, 1892) & 33,43 \\
\hline Lepidozikania cinerascens (Walker, 1855) * & 31 \\
\hline Leucanopsis acuta (Hampson, 1901) & $6,12,24,27,43,50$ \\
\hline Leucanopsis bactris (Sepp, [1847]) & 33,39 \\
\hline Leucanopsis calvona (Schaus, 1941) & 24 \\
\hline Leucanopsis coniota (Hampson, 1901) & 43 \\
\hline Leucanopsis fuscosa (Jones, 1908) * & 33 \\
\hline \multicolumn{2}{|l|}{ Leucanopsis leucanina (R. Felder \& } \\
\hline Rogenhofer, 1874) & $6,24,27,32,33,34,50,55$ \\
\hline Leucanopsis lineata (Schaus, 1894) & 1,6 \\
\hline Leucanopsis liparoides (Rothschild, 1909) & 39 \\
\hline $\begin{array}{l}\text { Leucanopsis mandus (Herrich-Schäffer, } \\
{[1853] \text { ) }}\end{array}$ & 43 \\
\hline Leucanopsis oruba (Schaus, 1892) & $6,18,24,33,43,50$ \\
\hline Leucanopsis pulverea (Schaus, 1896) & 22,33 \\
\hline Leucanopsis quadrata (Rothschild, 1910) & 43 \\
\hline Leucanopsis quanta (Schaus, 1896) & 27 \\
\hline Leucanopsis rhomboidea (Sepp, [1852]) * & 32 \\
\hline Leucanopsis sablona (Schaus, 1896) * & 33 \\
\hline Leucanopsis umbrosa (Hampson, 1901) & 12,24 \\
\hline Lophocampa arpi (Dognin, 1923) & 15,34 \\
\hline Lophocampa catenulata (Hübner, [1812]) & $\begin{array}{l}1,6,22,24,32,33,34 \\
35,50\end{array}$ \\
\hline Lophocampa citrina (Sepp, [1843]) & $6,12,33,49$ \\
\hline Lophocampa dinora (Schaus, 1924) & 43 \\
\hline Lophocampa romoloa (Schaus, 1933) & 43 \\
\hline Lophocampa texta (Herrich-Schäffer, [1855]) & $6,24,27,33,34,50,55$ \\
\hline Machadoia xanthosticta (Hampson, 1901) & $15,27,31,33,34,46,50$ \\
\hline Mazaeras conferta Walker, 1855 & $12,23,24,27,33,50$ \\
\hline Mazaeras francki (Schaus, 1899) & $12,22,31,33$ \\
\hline Mazaeras janeira (Schaus, 1892) & 6,27 \\
\hline Melese asana Druce, $1884 *$ & 31,47 \\
\hline Melese binotata (Walker, 1856) * & 22 \\
\hline Melese castrena Schaus, 1905 & $6,12,24,43,50$ \\
\hline Melese chozeba (Druce, 1884) & $6,12,24,50$ \\
\hline Munona iridescens Schaus, 1894 & $12,24,39,50$ \\
\hline Neidalia dulcicula Schaus, 1929 & 27 \\
\hline Neonerita dorsipuncta Hampson, 1901 & $6,12,22,24,31,33,47,50$ \\
\hline Neritos repanda Walker, 1856 & $6,12,24,27,33,34,50$ \\
\hline Opharus basalis Walker, 1856 & $6,15,24,31,33,43,47,50$ \\
\hline Opharus bimaculata (Dewitz, 1877) & $12,31,50$ \\
\hline Opharus flavimaculata Hampson, 1901 * & 15 \\
\hline Opharus intermedia Rothschild, 1909 & $34,43,50$ \\
\hline Opharus procroides Walker, 1855 & $6,12,15,24,27,31,50$ \\
\hline Opharus rema (Dognin, 1891) & $6,12,27,31,33,42,47,55$ \\
\hline Ormetica chrysomelas (Walker, 1856) & $\begin{array}{l}6,12,22,23,24,25,27 \\
29,31,50\end{array}$ \\
\hline Pareuchaetes aurata (Butler, 1875) & $6,22,24,27,31,33,47,50$ \\
\hline Pelochyta cinerea (Walker, 1855) & $\begin{array}{l}6,12,24,27,31,34,43 \\
46,50\end{array}$ \\
\hline Phaegoptera albimacula (Jones, 1908) & 27,50 \\
\hline Phaegoptera chorima Schaus, 1896 & $29,31,46,50$ \\
\hline $\begin{array}{l}\text { Phaegoptera flavopunctata Herrich- } \\
\text { Schäffer, [1855] }\end{array}$ & 43 \\
\hline $\begin{array}{l}\text { Phaegoptera flavostrigata Herrich-Schäffer, } \\
\text { [1855] }\end{array}$ & $6,34,43,50$ \\
\hline $\begin{array}{l}\text { Phaegoptera histrionica Herrich-Schäffer, } \\
\text { [1855] }\end{array}$ & 31,43 \\
\hline
\end{tabular}

Apêndice I. Continuação.

\begin{tabular}{ll}
\hline Espécies & $\begin{array}{l}\text { Localidades } \\
\text { (ver Tabela I) }\end{array}$ \\
\hline $\begin{array}{l}\text { Phaegoptera nexa Herrich-Schäffer, [1855] } \\
\text { Phaegoptera punctularis Herrich-Schäffer, }\end{array}$ & 43 \\
& 31,46 \\
[1855] * & 33 \\
Phaegoptera schaefferi Schaus, 1892 * & 43 \\
Phaegoptera superba (Druce, 1911) * & 33,35 \\
Rhipha flavoplaga (Schaus, 1905) & $6,27,55$ \\
Rhipha flavoplagiata (Rothschild, 1911) & 33 \\
Rhipha fulminans (Rothschild, 1916) & 33 \\
Rhipha mathildae (Köhler, 1924) * & $29,33,34,35,43,49$ \\
Rhipha subflammans (Rothschild, 1909) & 33 \\
Robinsonia praphaea Dognin, 1906 & $15,22,24,27,31,55$ \\
Romualdia elongata (Felder, 1874) & 12,31 \\
Sychesia coccina Jordan, 1916 * & $6,33,49$ \\
Sychesia dryas (Cramer, 1775) & $6,12,24,27,32,33,34$, \\
Symphlebia catenata (Schaus, 1905) & 49,50 \\
& $32,33,44$ \\
Symphlebia erratum (Schaus, 1933) * & 12 \\
Symphlebia indistincta (Rothschild, 1909) * & $3,43,46$ \\
Symphlebia lophocampoides Felder, 1874 & 31 \\
Symphlebia suanus (Druce, 1902) * & 31 \\
Tessella jorgenseni (Schaus, 1921) & 33 \\
Tessella sertata (Berg, 1882) & $6,24,31,34,36,50$ \\
Tessellarctia semivaria (Walker, 1856) & $6,15,22,27,31,33,34$, \\
& $50,51,55$ \\
Tessellota trifasciata (Burmeister, 1878) & 34 \\
Viviennea dolens (Druce, 1904) & $6,12,22,24,27,35,39$, \\
& 50
\end{tabular}

Viviennea flavicincta (Herrich-Schäffer, [1855])*

Xanthophaeina levis (Druce, 1899)

Subfamília Lithosiinae (27 espécies)

Tribo Cisthenini (13 espécies)

Cisthene rosacea (Schaus, 1896) 31, 32, 33, 47

Illice blanda Jones, 1914

Illice calochroma (Snellen, 1878)

Illice cryptopyra Hampson, 1903

Illice persimilis Hampson, 1903

Illice rhodocraspis Hampson, 1905

Lycomorphodes strigosa (Butler, 1877)

Lycomorphodes suspecta (Felder, 1875)

Odozana domina (Schaus, 1896)

Pronola magniplaga Schaus, 1899

Talara niveata Butler, 1878

Zonoda dives Schaus, 1896

Zonoda endoxantha (Hampson, 1903)

Tribo Eudesmini (3 espécies)

Eudesmia argentinensis (Rothschild, 1912)

Eudesmia ruficollis Donovan, 1798

Euryptidia univitta Hampson, 1900

Tribo Lithosiini (11 espécies)

Agylla argentea (Felder, 1874)

Agylla separata (Schaus, 1874)

Apistosia judas Hübner, 1818

Areva subfulgens (Schaus, 1896)

Diarhabdosia mandana Dyar, 1907

Lamprostola pascuala (Schaus, 1896)

Metalobosia cuprea (Schaus, 1896)

Metalobosia varda (Schaus, 1896)

Nodozana endoxantha Jones, 1908

Nodozana rhodosticta (Butler, 1878)

Trichomelia celenna Schaus, 1892
12,31

31,43

31,47

31

31,46

31

31

32

12,31

31,47

31,47

44

$12,31,32,47$

31,47

$9,16,21,54$

4

33

31

$31,33,46$

12

31

33

$12,31,32,47$

12,31

31

46

32,36

32

Recebido em 17/05/2008; aceito em 17/11/2008 